Review

\title{
Efficacy of tendon and nerve gliding exercises for carpal tunnel syndrome: a systematic review of randomized controlled trials
}

\author{
SANG-Dol KIM, RN, $\mathrm{PhD}^{1)}$ \\ 1) Department of Nursing, College of Health Science, Kangwon National University: 346 Hwangjo-gil, \\ Dogye-eup, Samcheok-si, Gangwon-do 245-907, Republic of Korea
}

\begin{abstract}
Purpose] The purpose of this study was to assess the quality of evidence for the efficacy of tendon and nerve gliding exercises in the management of carpal tunnel syndrome. [Subjects and Methods] Four electronic databases were searched to identify randomized controlled trials on the efficacy of tendon and nerve gliding exercises for carpal tunnel syndrome. Quality assessment was conducted using the Cochrane risk of bias tool. [Results] Four trials were identified and included in the review. The results of critical appraisal of quality ranged between low and moderate risk of bias. The available data could only be included as a narrative description. Symptom severity decreased and functional status improved with combined treatment, involving a tendon or nerve gliding exercise group plus conventional treatments, compared with the use of conventional treatments alone. [Conclusion] Evidence from 4 randomized controlled trials suggests that tendon and nerve gliding exercises, when combined with conventional treatments, may have a favorable effect in patients with carpal tunnel syndrome. However, further randomized controlled trials designed to assess the effect of tendon and nerve gliding exercises alone are required to investigate the hypothesis that such exercises alleviate carpal tunnel syndrome, and to confirm and further elucidate the efficacy of standardized physical exercise programs in patients with carpal tunnel syndrome.

Key words: Tendon and nerve gliding exercises, Carpal tunnel syndrome
\end{abstract}

(This article was submitted Mar. 23, 2015, and was accepted Apr. 24, 2015)

\section{INTRODUCTION}

Our hands have a variety of functions, including activities of daily living and job performance ${ }^{1)}$; these functions can be restricted by carpal tunnel syndrome (CTS), a compressive neuropathy of the median nerve that occurs within the carpal tunnel at the wrist ${ }^{2-5}$. The incidence of CTS is reported to be between $2.7 \%$ and $5.8 \%{ }^{6}$. The most common symptoms are pain and paresthesia of the fingers, which occur secondary to impairment of median nerve functions; CTS can have a negative effect on a client's quality of life ${ }^{7)}$. Conservative treatment options include splinting, specific exercises, paraffin therapy, medications, and therapeutic ultrasound. Of these conservative treatments, tendon and nerve gliding exercises are popular, and have been used since 1990 in the management of $\mathrm{CTS}^{5-14)}$. However, while evidence for the efficacy of tendon and nerve gliding exercises for CTS is emerging, the use of these exercises for the treatment of CTS remains controversial ${ }^{7,10,15)}$. It has been stated previously that support for the use of tendon and nerve gliding exercises

Corresponding author. Sang-Dol Kim (E-mail: nu11110@, kangwon.ac.kr)

(C2015 The Society of Physical Therapy Science. Published by IPEC Inc. This is an open-access article distributed under the terms of the Creative Commons Attribution Non-Commercial No Derivatives (by-ncnd) License $<$ http://creativecommons.org/licenses/by-nc-nd/3.0/>. in the treatment of CTS will require high-quality studies with rigorous methodological approaches ${ }^{7-9,12,16)}$. Therefore, the aim of this review was to assess the quality of evidence for the efficacy of tendon and nerve gliding exercises in the management of CTS.

\section{SUBJECTS AND METHODS}

This review was planned and conducted in accordance with Preferred Reporting Items for Systematic Reviews and Meta-Analyses (PRISMA) guidelines ${ }^{16}$ ) and Consolidated Standards of Reporting Trials (CONSORT) guidelines for reporting parallel group randomized trials ${ }^{17)}$. Four electronic databases (Cumulative Index to Nursing and Allied Health Literature (CINAHL), the Cochrane Library, Embase, and PubMed) were searched to identify randomized controlled trials (RCTs) published in the period between 1963 and January 2015. The search terms were carpal tunnel syndrome AND tendon and nerve gliding exercises OR tendon gliding exercises OR nerve gliding exercises. All potentially eligible studies were retrieved, and the full-text articles were reviewed to determine whether they met the following selection criteria.

To be eligible for inclusion, studies had to meet the following conditions: 1) Participants in the trials had to be diagnosed with CTS. 2) The studies had to be RCTs that used tendon and nerve gliding exercises as an intervention to reduce symptoms associated with CTS compared with 
no tendon and nerve gliding exercises. 3) The outcome measures had to be symptom severity and functional status. Quality assessment of included articles was conducted using a critical appraisal tool (the Cochrane Collaboration's risk of bias tool), as recommended in the Cochrane Handbook for Systematic Reviews of Interventions ${ }^{18)}$. The Cochrane risk of bias tool is a 6-item list designed to assess sequence generation, allocation concealment, blinding, incomplete outcome data, selective outcome reporting, and other potential sources of bias. Each item was rated as "yes", "no", or "unclear". According to the Cochrane Handbook, there are 3 levels of evidence: A, B, and C. The level assigned to a study gives an indication of the quality of the trial ${ }^{19)}$. If the study design fully met all of the preceding 6 criteria, its level was considered to be A (low risk of bias). A study was assigned to be the B level when one or more criteria were partly met. If one or more criteria were not met, the study was assigned to be the $\mathrm{C}$ level, implying a high risk of bias ${ }^{19)}$. Any study assigned to the $\mathrm{C}$ level was eliminated from this review.

\section{RESULTS}

A total of 164 articles related to the search terms were screened. Among them, potentially relevant trials were identified in the CHINAL ( $\mathrm{n}=7)$, Cochrane library $(\mathrm{n}=14)$, Embase $(n=25)$, and PubMed $(n=21)$ databases. After the titles of the articles were retrieved, a total of 48 studies were excluded due to retrieval of duplicate articles, study designs other than RCTs (case studies, commentaries, or review articles), or a lack of target concepts in the article (i.e., no CTS). The abstracts of the remaining 19 articles were retrieved. After assessing the abstracts, 4 studies were excluded on the basis of an absence of tendon and nerve gliding exercises; thus, a total of 15 potentially relevant trials were identified in the search; all 15 articles were retrieved for evaluation of their full texts. After assessment of the full articles, 11 studies were excluded; 8 studies did not involve a randomized trial, and 3 studies did not contain the full texts of the RCTs. The literature retrieval process is depicted in Fig. 1. Characteristics of the included studies are presented in Table 1.

With regards to country of origin, 2 RCTs were conducted in Turkey ${ }^{6,11)}$, 1 was conducted in the USA ${ }^{8)}$, and 1 was conducted in Taiwan ${ }^{7)}$. Studies were conducted at the following centers: the University of Pittsburgh Medical Center's Orthopedic Outpatient Hand Clinic, the department of physical medicine and rehabilitation of a community hospital, the Outpatient Clinic at the Istanbul Physical Medicine and Rehabilitation Training and Research Hospital, and the Department of Physical Medicine and Rehabilitation and the Department of Neurology of Dokuz Eylül University. Sample sizes in the 4 studies ranged from 36 to 111 participants and totaled 261 participants overall, with 243 being female and 18 being male. The mean age of participants reported in the different studies ranged from $49.1-51.9$ years. The overall median age of participants was 50.3 years.

The 4 RCTs identified in this review were analyzed. Types of intervention were heterogeneous among the studies: 1 RCT used paraffin therapy plus splints plus tendon gliding exercises in group 1, paraffin therapy plus splints plus nerve gliding exercises in group 2, and paraffin therapy plus splints

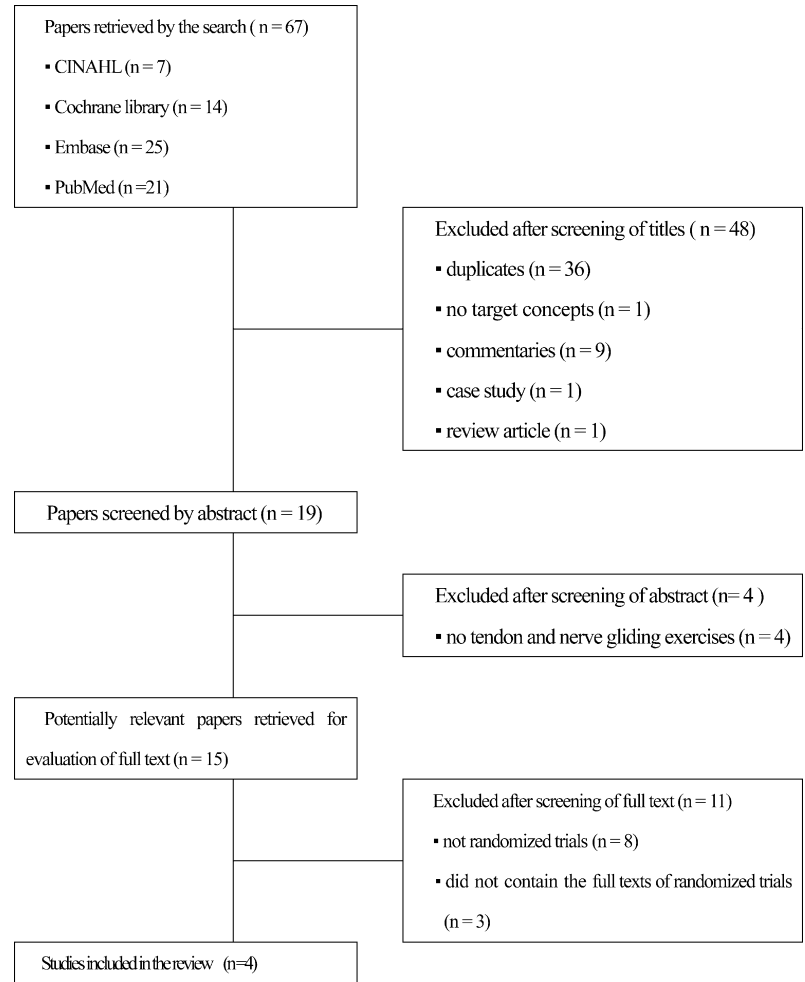

Fig. 1. Flowchart for the included studies from the literature searches

in group 3; another RCT used standard conservative treatment (SCT) in group 1, SCT plus tendon and nerve gliding exercises in group 2, and tendon and nerve gliding exercises in group 3; another RCT used neutral wrist/metacarpophalangeal (NW/MCP) splints in group 1, NW/MCP splints plus tendon and nerve gliding exercises in group 2, wrist cock-up (WCU) splints in group 3, and WCU splints plus tendon and nerve gliding exercises in group 4; and the remaining RCT used neutral volar wrist splints in group 1 and neutral volar wrist splints plus tendon gliding exercises in group 2 .

The Intervention lengths of the 4 trials ranged from 4 weeks to 11 months. Interventions were performed under the guidance of physiotherapists, and a brochure describing the tendon and nerve exercises was provided to all patients.

All 4 trials reported an effect on CTS. The available data could only be included as a narrative description. Symptom severity and functional statuses were improved in groups that received combined treatment with tendon and nerve gliding exercises and conventional treatments compared with the groups that received conventional treatments alone. No study reports included data relating to adverse effects of treatment (Table 1).

The risks of bias in the 4 studies were low or moderate.

\section{DISCUSSION}

The purpose of this review was to assess the evidence for the efficacy of tendon and nerve gliding exercises for CTS when compared with other treatments. A meta-analysis combining the results from all the trials was not feasible 


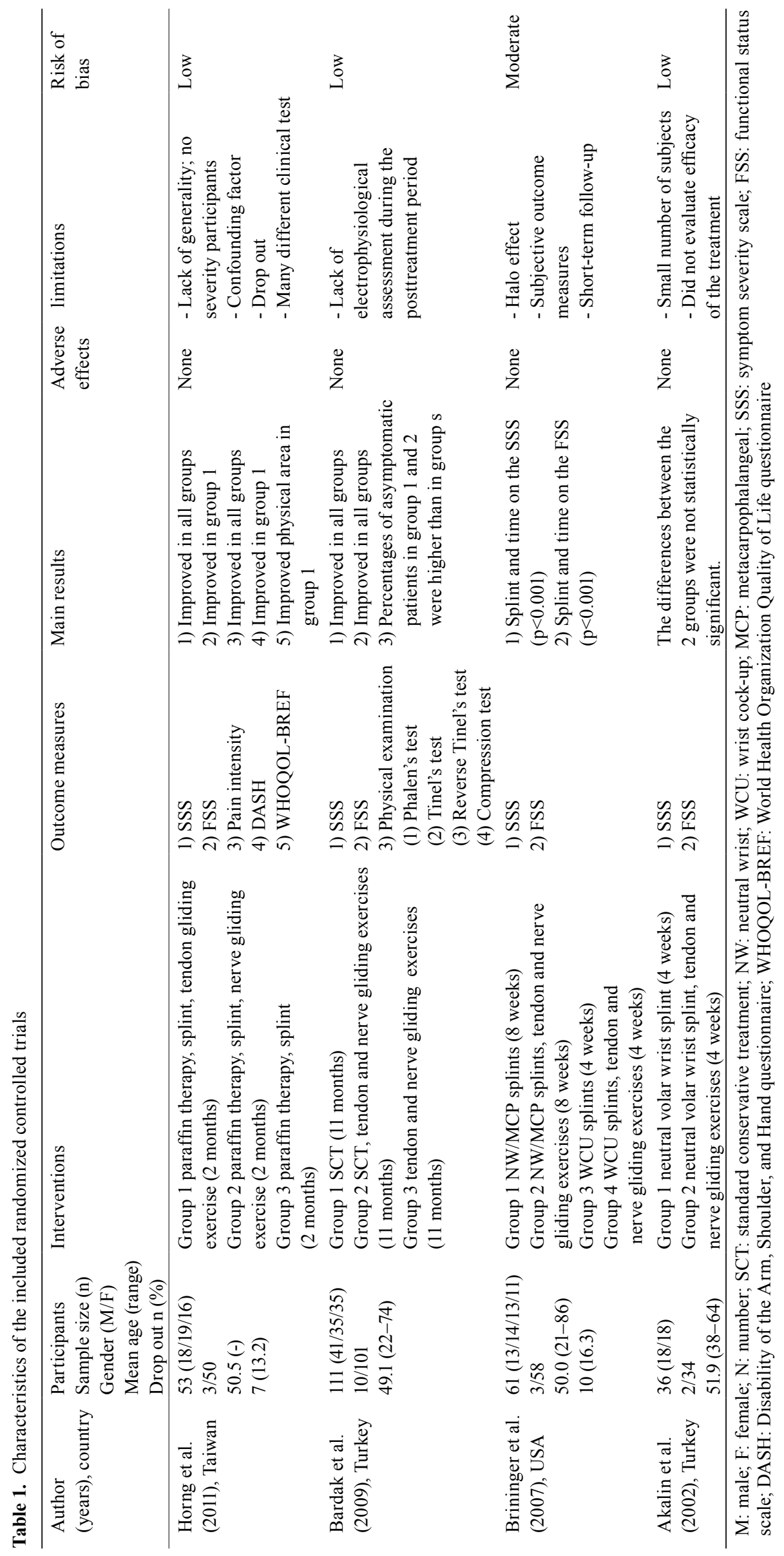


due to the heterogeneity of the identified studies. A total of 4 RCTs were identified and included in this review. The interventions in all studies included tendon and nerve gliding exercises to manage CTS.

All 4 trials reported a significant improvement in CTSrelated symptom severity and functional status in all groups. However, the efficacy of the tendon and nerve gliding exercises alone could not be identified because the 4 trials involved different combinations of tendon and nerve gliding exercises with standard conservative treatments that included splints, paraffin therapy, and other exercises. Additional RCTs are required to provide evidence for the efficacy of tendon and nerve gliding exercises alone as a management option for CTS.

The quality ratings of the trials included in this review indicated low or moderate risks of bias. Two trials did not mention blinding, which may have led to biased results. Overall, no strong conclusions can be made due to the small number of included trials and other methodological considerations.

The use of rigorous methodological criteria is a strength of this systematic review. Among the included studies major strengths included the use of randomization, and the quality of the measurement tools utilized. There are no reported adverse effects associated with the use of tendon and nerve gliding exercises in the treatment of CTS.

Limitations of the included studies were also identified. Firstly, the generalizability of results is limited because most of the participants had mild or moderate symptoms. Therefore, caution should be exercised when attempting to apply these findings to patients with more severe symptoms. Secondly, there were issues with blinding; the persons who administered the treatment and evaluated outcomes were not blinded to the subjects' group assignments. For strict and rigorous methodological research, blinding is necessary because it may reduce the risk of ascertainment bias. Of the 4 RCTs included in this review, two articles involved single blinding, and none of the RCTs included in this review involved double blinding. However, blinding is not easy to implement with interventions such as physical exercises; therefore post hoc research should be processed with strict blinding. Finally, none of the RCTs used tendon and nerve gliding exercises alone as a treatment for CTS. This may have led to difficulties in interpretation and identification of the efficacy of the tendon and nerve gliding exercises in the management of CTS. All 4 RCTs combined tendon and nerve gliding exercises with standard conservative treatments.

In conclusion, although this review included only a limited number of small trials, it had several strengths, such as the inclusion of only RCTs and the use of outcome measures that are reliable, valid, and commonly used in clinics. However further rigorous methodological and high-quality RCTs are needed to confirm and further understand the efficacy of standardized tendon and nerve gliding exercises as an intervention to control symptom severity and improve functional status in CTS. Future studies need to take into consideration the value of larger sample sizes and ensure a more strenuous and rigorous adherence to the exercise protocols.

\section{REFERENCES}

1) Jung HY, Chang M, Kim KM, et al.: Effect of wrist joint restriction on forearm and shoulder movement during upper extremity functional activities. J Phys Ther Sci, 2013, 25: 1411-1414. [Medline] [CrossRef]

2) Oskouei AE, Talebi GA, Shakouri SK, et al.: Effects of neuromobilization maneuver on clinical and electrophysiological measures of patients with carpal tunnel syndrome. J Phys Ther Sci, 2014, 26: 1017-1022. [Medline] [CrossRef]

3) Ashworth NL: Carpal tunnel syndrome. BMJ Clin Evid, 2011, 2011: 1114 [Medline]

4) Burke FD, Ellis J, McKenna H, et al.: Primary care management of carpal tunnel syndrome. Postgrad Med J, 2003, 79: 433-437. [Medline] [CrossRef]

5) Baysal O, Altay Z, Ozcan C, et al.: Comparison of three conservative treatment protocols in carpal tunnel syndrome. Int J Clin Pract, 2006, 60: 820-828. [Medline] [CrossRef]

6) Bardak AN, Alp M, Erhan B, et al.: Evaluation of the clinical efficacy of conservative treatment in the management of carpal tunnel syndrome. Adv Ther, 2009, 26: 107-116. [CrossRef] [Medline]

7) Horng YS, Hsieh SF, Tu YK, et al.: The comparative effectiveness of tendon and nerve gliding exercises in patients with carpal tunnel syndrome: a randomized trial. Am J Phys Med Rehabil, 2011, 90: 435-442. [CrossRef] [Medline]

8) Brininger TL, Rogers JC, Holm MB, et al.: Efficacy of a fabricated customized splint and tendon and nerve gliding exercises for the treatment of carpal tunnel syndrome: a randomized controlled trial. Arch Phys Med Rehabil, 2007, 88: 1429-1435. [Medline] [CrossRef]

9) Duymaz T, Sindel D, Kesiktas N, et al.: Efficacy of some combined conservative methods in the treatment of carpal tunnel syndrome: a randomized controlled clinical and electrophysiological trial. TURK J Rheumatol, 2012, 27: 38-46. [CrossRef]

10) Horng YS, Hsieh SF, Lin MC, et al.: Ultrasonographic median nerve changes under tendon gliding exercise in patients with carpal tunnel syndrome and healthy controls. J Hand Ther, 2014, 27: 317-323, quiz 324. [CrossRef] [Medline]

11) Akalin E, El O, Peker O, et al.: Treatment of carpal tunnel syndrome with nerve and tendon gliding exercises. Am J Phys Med Rehabil, 2002, 81: 108-113. [Medline] [CrossRef]

12) Incebiyik S, Boyaci A, Tutoglu A, et al.: Short-term effectiveness of shortwave diathermy treatment on pain, clinical symptoms, and hand function in patients with mild or moderate idiopathic carpal tunnel syndrome. $\mathrm{J}$ Back Musculoskelet Rehabil, 2015, 28: 221-228.

13) Goodyear-Smith F, Arroll B: What can family physicians offer patients with carpal tunnel syndrome other than surgery? A systematic review of nonsurgical management. Ann Fam Med, 2004, 2: 267-273. [Medline] [CrossRef]

14) Rozmaryn LM, Dovelle S, Rothman ER, et al.: Nerve and tendon gliding exercises and the conservative management of carpal tunnel syndrome. J Hand Ther, 1998, 11: 171-179. [Medline] [CrossRef]

15) Martin BI, Levenson LM, Hollingworth W, et al.: Randomized clinical trial of surgery versus conservative therapy for carpal tunnel syndrome [ISRCTN84286481]. BMC Musculoskelet Disord, 2005, 6: 2 [CrossRef]. [Medline]

16) Moher D, Liberati A, Tetzlaff J, et al. PRISMA Group: Preferred reporting items for systematic reviews and meta-analyses: the PRISMA statement. Ann Intern Med, 2009, 151: 264-269, W64. [Medline] [CrossRef]

17) Schulz KF, Altman DG, Moher D, CONSORT Group: CONSORT 2010 Statement: updated guidelines for reporting parallel group randomised trials. J Clin Epidemiol, 2010, 63: 834-840. [Medline] [CrossRef]

18) Higgins JP, Green S: Cochrane Handbook for Systematic Reviews of Interventions. Version 5.1.0 (updated March 2011) ed. The Cochrane Collaboration, 2011.

19) Yang LH, Duan PB, Du SZ, et al.: Efficacy of auriculotherapy for constipation in adults: a systematic review and meta-analysis of randomized controlled trials. J Altern Complement Med, 2014, 20: 590-605. [Medline] [CrossRef] 